\title{
Construction and characterisation of a complete reverse genetics system of dengue virus type 3
}

\author{
Jefferson José da Silva Santos ${ }^{1}$, Marli Tenório Cordeiro, ${ }^{1,2}$, Giovani Rota Bertani ${ }^{3}$, \\ Ernesto Torres de Azevedo Marques ${ }^{1,4}$, Laura Helena Vega Gonzales Gil1/+
}

\author{
'Departamento de Virologia e Terapia Experimental, Centro de Pesquisas Aggeu Magalhães-Fiocruz, Recife, PE, Brasil \\ ${ }^{2}$ Laboratório Central de Saúde Pública, Departamento de Saúde do Estado de Pernambuco, Recife, PE, Brasil \\ ${ }^{3}$ Departamento de Bioquímica, Universidade Federal de Pernambuco, Recife, PE, Brasil ${ }^{4}$ Department of Infectious Diseases \\ and Microbiology, Center for Vaccine Research, University of Pittsburgh, Pittsburgh, PA, USA
}

\begin{abstract}
Dengue virulence and fitness are important factors that determine disease outcome. However, dengue virus (DENV) molecular biology and pathogenesis are not completely elucidated. New insights on those mechanisms have been facilitated by the development of reverse genetic systems in the past decades. Unfortunately, instability of flavivirus genomes cloned in Escherichia coli has been a major problem in these systems. Here, we describe the development of a complete reverse genetics system, based on the construction of an infectious clone and replicon for a low passage DENV-3 genotype III of a clinical isolate. Both constructs were assembled into a newly designed yeast-E. coli shuttle vector by homologous recombination technique and propagated in yeast to prevent any possible genome instability in E. coli. RNA transcripts derived from the infectious clone are infectious upon transfection into $B H K-21$ cells even after repeated passages of the plasmid in yeast. Transcript-derived DENV-3 exhibited growth kinetics, focus formation size comparable to original DENV-3 in mosquito C6/36 cell culture. In vitro characterisation of DENV-3 replicon confirmed its identity and ability to replicate transiently in BHK-21 cells. The reverse genetics system reported here is a valuable tool that will facilitate further molecular studies in DENV replication, virus attenuation and pathogenesis.
\end{abstract}

Key words: reverse genetics - dengue virus - molecular cloning

Dengue virus (DENV) is an arthropod-borne virus classified in the Flavivirus genus with four serotypes (DENV-1-4) responsible for a wide spectrum of clinical outcomes around the world. Other medical relevant viruses in this genus are yellow fever virus (YFV), West Nile virus, Japanese encephalitis virus and tick-borne encephalitis virus (Monath 1994, Lindenbach et al. 2007). The flavivirus genome consists of a 5' m7 G-capped single-stranded positive RNA of approximately $11 \mathrm{~Kb}$ in length, containing a single open reading frame (ORF) flanked by untranslated regions [5'-untranslated region (UTR) and 3'-UTR]. Release of virus RNA in susceptible cells leads to the translation of the ORF producing a polyprotein that is co- and posttranslationally processed to generate three structural $(\mathrm{C}, \mathrm{prM}$ and $\mathrm{E})$ and seven nonstructural (NS1, NS2a, NS2b, NS3, NS4a, NS4b and NS5) proteins (Chambers et al. 1990, Clyde et al. 2006, Lindenbach et al. 2007, Fernandez-Garcia et al. 2009).

The use of reverse genetics systems has dramatically changed the study of RNA virus, allowing a wide range

doi: $10.1590 / 0074-0276130298$

Financial support: FIOCRUZ (Papes V), FACEPE (APQ-0519-

2.12/06), CNPq (470892/2007-2)

JJSS receives a graduate scholarship from the $\mathrm{CNPq}$

+ Corresponding author: laura@cpqam.fiocruz.br

Received 4 June 2013

Accepted 12 September 2013 of genetic manipulations in the cloned genome (Ruggli \& Rice 1999, Bouloy \& Flick 2009). The flavivirus infectious clone methodology was first established for YFV (Rice et al. 1989) and since then it has provided valuable insights in genome replication (Alvarez et al. 2005), virus pathogenesis (Liu et al. 2006), vaccine development (Guy et al. 2008) and antiviral research (Puig-Basagoiti et al. 2005). Infectious cDNA clones have been successfully developed for other flaviviruses (Yamshchikov et al. 2001, Pu et al. 2011) and DENV (Lai et al. 1991, Kapoor et al. 1995, Puri et al. 2000, Blaney Jr et al. 2004). An alternative approach developed to study flaviviruses involves the construction of subgenomic replicons derived from the corresponding parental infectious clone (Jones et al. 2005, Alcaraz-Estrada et al. 2010). Lacking one or more structural proteins, replicons are capable of autonomous replication in susceptible cells even though they are unable to produce virus particles (Khromykh \& Westaway 1997, Jones et al. 2005, Widman et al. 2008). Yet, most of these systems are based on established strains or clinical isolates adapted to growth in cultured cells after several passages and only few studies have focused in low passage virus strains (Kurosu et al. 2010), which are important assets in deciphering key elements in virus pathogenesis and virulence determinants (Ubol et al. 2008, Tuiskunen et al. 2011).

In an ideal reverse genetics system, cloned flavivirus genomes should be stably propagated. Unfortunately, instability of full-length cDNA clones in Escherichia coli remains a major hold back to development of these systems (Polo et al. 1997, Ruggli \& Rice 1999). The 
genome sequence at the E/NS1 region or an expressed product from that region appears to be associated with this toxicity (Polo et al. 1997, Blaney Jr et al. 2004), but others regions might be implicated as well $(\mathrm{Pu}$ et al. 2011). Several approaches have been developed and evaluated to overcome genome instability of flavivirus infectious cDNA clones, including: the incorporation of a linker containing redundant termination codons in the genome (Blaney Jr et al. 2004), the use of an in vitro ligation strategy derived from two plasmids (Kapoor et al. 1995), the use of low-copy number vectors (Bredenbeek et al. 2003) or bacterial artificial chromosomes (BACs) (Suzuki et al. 2007), the use of different E. coli strains (Sriburi et al. 2001) or the cloning in yeast cells (Polo et al. 1997). More recently, investigators were able to surpass genome instability by introducing silent mutations in cryptic promoters in the viral genome to lower $E$. coli promoter activity ( $\mathrm{Pu}$ et al. 2011). In another strategy, DENV-3 genome was cloned as four segmented, sequential fragments and directionally in vitro ligated to create a full-length infectious clone. While novel to flaviviruses, this approach have been effectively used in coronavirus research (Messer et al. 2012).

In this study, we report the successful development of a complete reverse genetics system, based on the construction of an infectious clone and a replicon for a low passage DENV-3 clinical isolate. Using homologous recombination in yeast and a newly designed vector, we were able to overcome the inherent instability of flavivirus genome observed in E. coli.

\section{SUBJECTS, MATERIALS AND METHODS}

Cell lines and DENV-3 strain - BHK-21 cells were propagated at $37^{\circ} \mathrm{C}$ in a humidified incubator with $5 \%$ $\mathrm{CO}_{2}$ in minimum essential medium supplemented with $10 \%$ foetal bovine serum (FBS). Mosquito C6/36 cells were cultured at $30^{\circ} \mathrm{C}$ in Leibovitz's medium (L-15) with $5 \%$ FBS. Both growth media were supplemented with $1 \%$ antibiotic solution (containing penicillin and streptomycin). 95016/BR-PE/02 strain is a DENV-3 clinical sample isolated from an 11-year-old female patient during the DENV-3 epidemics in the state of Pernambuco, Brazil in 2002 (Cordeiro et al. 2007). The patient presented typical symptoms of dengue fever. Virus was isolated in mosquito C6/36 cells (passage 1) and virus serotype confirmed by reverse transcription-polymerase chain reaction (RT-PCR). Virus stocks were produced in mosquito $\mathrm{C} 6 / 36$ cell cultures and stored at $-70^{\circ} \mathrm{C}$. The infectious clone and replicon described herein were derived from passage 3 in insect cells.

E. coli strain, yeast strains and yeast transformation Electrocompetent ElectroMAX DH10B E. coli cells were acquired from Invitrogen. Saccharomyces cerevisiae RFY206 (MATa trp14::hisG his34200 ura3-52 lys24201 leu2-3) strain (Finley Jr \& Brent 1994) was grown in YPD media and made competent by lithium acetate treatment (Sambrook \& Russell 2001). S. cerevisiae YPH252 (MATa ura3-52 lys2-801 ade2-101 trp1-41 his3-4200 leu241) strain (Sikorski \& Hieter 1989) was purchased from ATCC. YPH252 transformation was carried out by electroporation as previously described (Meilhoc et al. 1990).
Plasmid constructions - Plasmid pSVJS01, a newly designed shuttle vector, was constructed as follows: a 2-kb fragment was amplified by PCR from pRS414 (ATCC). It contains necessary elements to allow replication (CEN6/ARS H1) and selection (TRP1) in yeast. The 5'-end primer used on this PCR reaction included a $\mathrm{T} 7$ promoter recognition site and a multiple cloning site (MCS). To assemble pSVJS01, the 2-kb fragment was inserted into NotI-linearised pBeloBAC11 vector (New England Biolabs) by homologous recombination (Polo et al. 1997, Gibson 2009) in RFY206 yeast strain. Recombinant clones were selected in drop-out YNB agar plates lacking tryptophan. Purified plasmid DNA was amplified in DH10B E. coli strain in selective medium and analysed by restriction pattern on agarose gel electrophoresis.

DENV-3 infectious clone was constructed as illustrated in Fig. 1. DENV-3 genome was amplified in five overlapping subgenomic fragments (Table) using the KlenTaq LA polymerase mix (Clonetech), a high fidelity DNA polymerase enzymes mix. The first fragment (F1) was amplified using primers pSVJS01-F and DENV-31860-R. The forward primer used on this PCR reaction included an RsrII restriction site, a T7 promoter recognition site and an additional $\mathrm{G}$ immediately before the first base of DENV-3 genome. Fragment F2 was amplified using primers DENV-3-1141-F and DENV-3-3521-R. This reaction used as template a RT product derived from a DENV-3 infectious clone recovered by in vitro ligation (JJS Santos \& LHVG Gil, unpublished observations) and introduced a unique BamHI site into the DENV-3 infectious clone sequence reported on this paper. The third fragment (F3) was amplified with primers DENV-3-3481-F and DENV-3-4570-R while primer set DENV-3-4370-F and DENV-3-6700-R was used to amplify fragment F4. The last fragment (F5) was amplified using primers DENV-3-6521-F and pSVJS01-R.

DENV-3 replicon was generated by a PCR-mediated deletion, eliminating almost the entire structural coding region [from nucleotide (nt) 454-nt 2336)], as depicted in Fig. 2, and cloned as a set of five fragments (Table), three of which have been previously described (F3, F4 and F5). The first fragment (RF1) was amplified using primers pSVJS01-F and rep-prM-BamHI-E-R, generating a $0.5-\mathrm{Kb}$ PCR product that retained the first six codons of prM. The second fragment (RF2) was amplified with primers rep-prM-BamHI-E-F and DENV-3521-R, producing a $1.3-\mathrm{Kb}$ PCR product carrying the last 26 codons of E protein. Primers rep-prM-BamHI-E-F and rep-prM-BamHI-E-R were engineered to insert a single BamHI site in the DENV-3 replicon sequence. Both constructs were assembled into NotI-linearised pSVJS01 vector by homologous recombination (Polo et al. 1997, Gibson 2009) in YPH252 yeast strain. Recombinant clones were selected in drop-out YNB agar plates, and then screened by PCR and DNA sequencing. Functional clones were maintained in yeast to prevent any instability of sequences in bacteria. Genome sequence was deposited in the GenBank under the accession KC425219.

In vitro transcription and transfection - Full-length PCR product from DENV-3 infectious clone was purified by phenol-chloroform extraction, followed by etha- 
nol precipitation. Full-length RNA transcripts were in vitro synthesised using the MEGAScript T7 kit (Ambion) with the addition of a 7-methyl-guanosine cap ana$\log$ (Ambion), following manufacturer's instructions. An aliquot from the reaction was analysed by formaldehyde-agarose gel electrophoresis and RNA transcripts were used without further processing. Full-length RNA transcripts were introduced into BHK-21 cells by electroporation using the ECM 830 electro cell manipulator (BTX Harvard Apparatus). 2 x $10^{6}$ BHK-21 cells were trypsinised and washed twice with serum-free medium, resuspended in $100 \mu \mathrm{L}$ Cytomix solution (Ansari et al. 2004) and mixed with $5 \mu \mathrm{g}$ of transcribed RNA. Cells were transferred to an ice-cold electroporation cuvette with $0.2-\mathrm{cm}$ gap. Electroporation was performed with two pulses of $100 \mu \mathrm{s}$ at $1,200 \mathrm{~V}$ separated by $1 \mathrm{~s}$ interval. As negative control, BHK-21 cells were transfected under the same conditions except no RNA was added (mock). After electroporation, cells were allowed to recover for $10 \mathrm{~min}$ at room temperature and resuspended in complete growth medium.

Immunofluorescence assay (IFA) - To detect DENV-3 virus antigen, transfected cells were seeded onto glass coverslips. At different time points, RNA-transfected cells in glass coverslips were rinsed with phosphate buffered saline (PBS), fixed with $50 \%$ cold acetone $(\mathrm{v} / \mathrm{v})$ in PBS for $5 \mathrm{~min}$ at $4^{\circ} \mathrm{C}$ and air-dried. Fixed cells were incubated with a 1:100 dilution of a polyclonal hyperimmune mouse ascitic fluid (HMAF) for $1 \mathrm{~h}$ at $37^{\circ} \mathrm{C}$.
This HMAF was raised against group B flaviviruses, including YFV and the four DENV serotypes. While its reactivity has not been fully tested, this HMAF reacts predominantly to E protein. After incubation with primary antibody, cells were rinsed twice with PBS and incubated for $1 \mathrm{~h}$ at $37^{\circ} \mathrm{C}$ with a 1:100 dilution of fluorescein isothiocyanate (FITC)-conjugated anti-mouse IgG antibody (Sigma-Aldrich), developed in goat. Then, cells were rinsed twice with PBS and the nuclei stained with a Hoechst 33258 solution (Sigma-Aldrich). Prior visualisation by fluorescent microscopy, cell were washed twice with $\mathrm{ddH}_{2} 0$, air-dried and mounted. Immunofluorescence analyses were carried out on a DMI 4000B inverted microscope (Leica).

Stability of DENV-3 infectious clone in yeast - To evaluate genome stability, cloned DENV-3 infectious clone was repeatedly propagated in yeast. Recombinant clones were grown in drop-out YNB agar plates at $30^{\circ} \mathrm{C}$ for three days. Grown colonies were streaked out in fresh selective agar plates and incubated at $30^{\circ} \mathrm{C}$ for other three days. After 10 consecutive passages, plasmid DNA was purified and genome integrity was assessed by DNA sequencing and virus rescue.

$D E N V-3$ replicon $R N A$ transcription and transfection - The entire DENV-3 replicon sequence was amplified by a high-fidelity full-length PCR. The PCR product was purified by phenol-chloroform extraction and ethanol precipitation and used as a template for in vitro transcription. Replicon transcripts were synthesised using the MEGA-

DENV-3 genome $10,707 \mathrm{nt}$

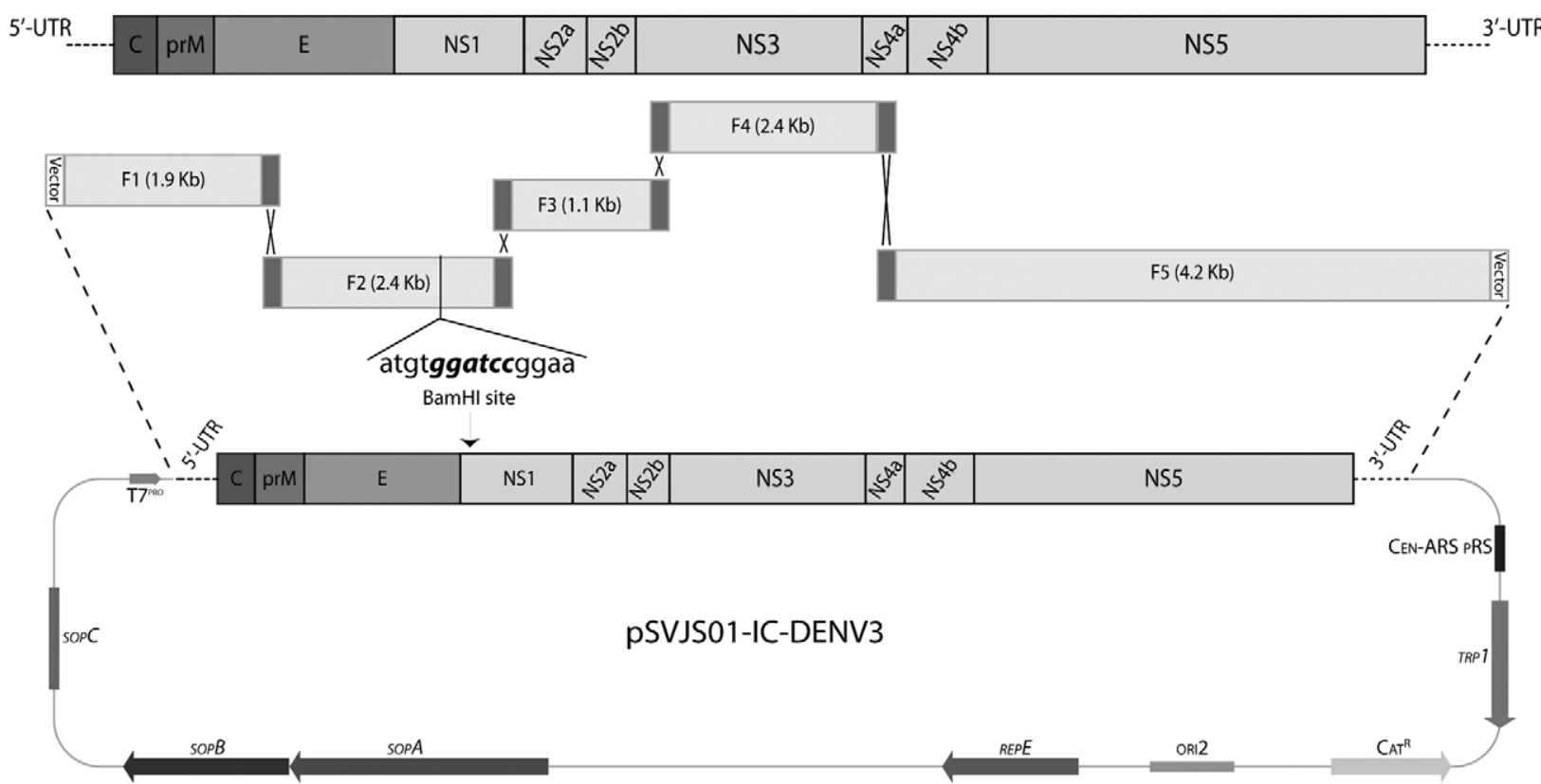

Fig. 1: schematic diagram for the construction of a full-length dengue virus type 3 (DENV-3) infectious clone with the yeast shuttle vector pSVJS01. DENV-3 genome was amplified in five overlapping subgenomic fragments: F1, F2, F3, F4 and F5. Full-length DENV-3 infectious clone was assembled into pSVJS01 vector by homologous recombination, generating pSVJS01-IC-DENV-3. A single BamHI site was engineered and incorporated into DENV-3 infectious clone sequence. Recombination sites (X) are indicated. NS: nonstructural; nt: nucleotide; UTR: untranslated region. 


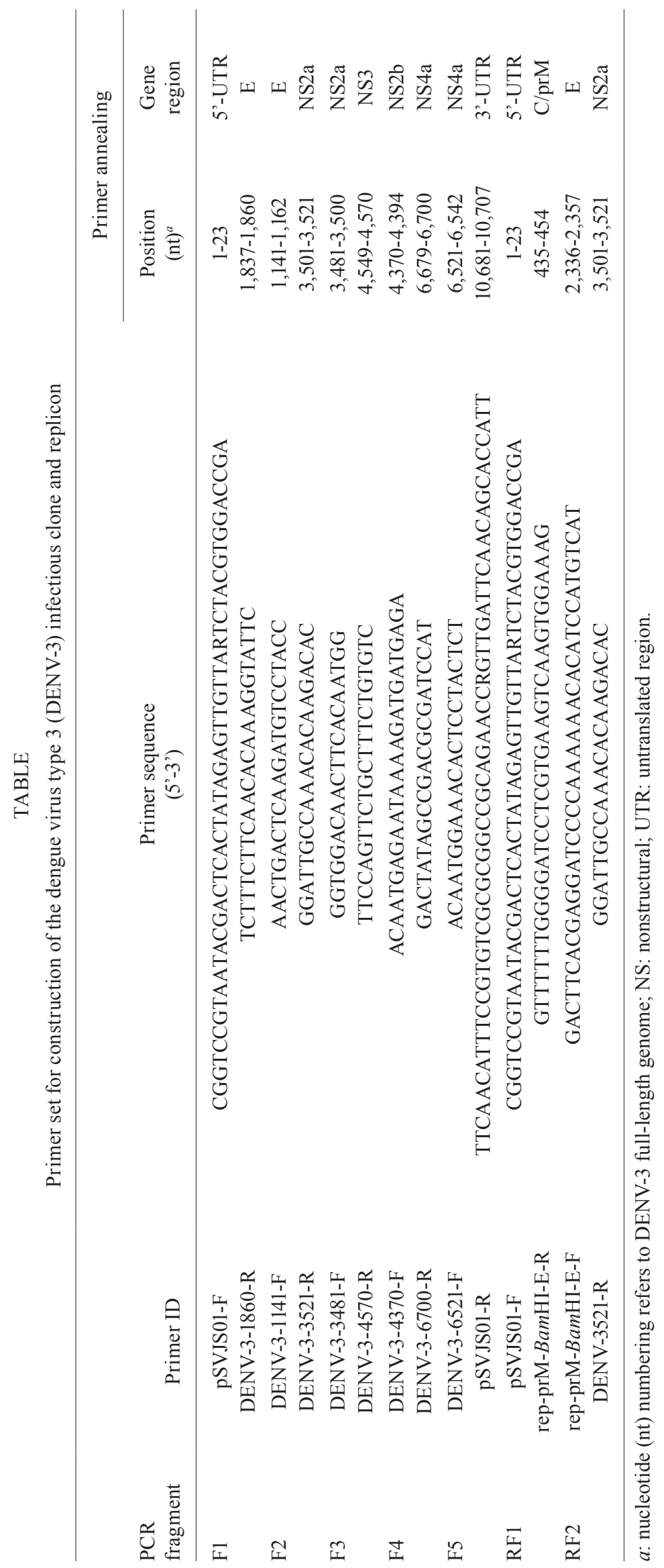


script T7 kit (Ambion) with the addition of a 7-methyl-guanosine cap analog (Ambion). RNA transcripts were electroporated into BHK-21 cells, as previously described.

DENV-3 replicon transient replication assessed by RT-PCR - At different time points, total RNA was purified from BHK-21 cells transfected with DENV-3 replicon using the Invisorb Spin Cell RNA mini kit (Invitek), according to manufacturer's instructions. Total RNA isolation from mock-transfected BHK-21 cells was used as negative control. Quantified RNA preparations served as templates to RT using SuperScript III Reverse Transcriptase (Invitrogen) with reverse primer DENV3-3521-R (5'-ggattgccaaacacaagacac-3'), following the manufacturer's protocol. Standard PCR amplifications were carried out using RT products as templates and specific primers: DENV-3-1-F (5'-agttgttagtctacgtggaccg-3') and DENV-3-3521-R (Table). RT-PCR products were analysed on agarose gel electrophoresis.

Virus titration and growth curves - Virus titration was determined by focus-forming essay in mosquito C6/36 cells, as described previously (Das et al. 2007) with modifications. Briefly, $2 \times 10^{5}$ cells per well were seed in 24-well cell culture dishes $48 \mathrm{~h}$ prior to the assay was conducted. Cell monolayers were incubated at $30^{\circ} \mathrm{C}$ with $0.2 \mathrm{~mL}$ of serial dilutions of each virus. After removing the inocula, cells were covered with $1 \mathrm{~mL}$ of L-15 overlay medium, containing $1 \%$ carboxymethyl cellulose, 2\% FBS and 1\% antibiotic solution and incubated at $30^{\circ} \mathrm{C}$. On day 5 , overlaid medium was removed. Cells were fixed with $30 \%$ cold acetone (v/v) in PBS for $13 \mathrm{~min}$ at room temperature and washed once with PBS. Fixed cells were incubated for $1 \mathrm{~h}$ at $37^{\circ} \mathrm{C}$ with a HMAF diluted 1:100 in ligation buffer. Cells were rinsed with wash buffer and incubated with a 1:500 dilution in ligation buffer of a horseradish peroxidase-conjugated recombinant protein $\mathrm{G}$ (Invitrogen) for $1 \mathrm{~h}$ at $37^{\circ} \mathrm{C}$. Cells were rinsed with wash buffer and foci developed by adding 3-amino-9-ethylcarbazole substrate buffer. Foci were counted and virus titres, expressed in focus-forming unit per $\mathrm{mL}$, determined by standard methods. For growth curves, $5 \times 10^{5}$ mosquito $\mathrm{C} 6 / 36$ cells per well in a 24-well cell culture dishes were infected in duplicate with wild-type DENV-3 (WT-DENV-3) and transcriptderived DENV-3 at a multiplicity of infection (MOI) of 0.1 . Cells were incubated at $30^{\circ} \mathrm{C}$ for $1 \mathrm{~h}$. After inocula removal, cells were washed twice with PBS and $1 \mathrm{~mL}$ of growth medium was added to each well. Aliquots of the supernatant of infected cells were removed daily and stored at $-70^{\circ} \mathrm{C}$. DENV-3 titres in each sample were determined by titration in mosquito $\mathrm{C} 6 / 36$ cells.

Statistical analysis - Statistical analyses were performed using the GraphPad Prism 4 for Macintosh (GraphPad Software, Inc). Growth kinetics differences were analysed by a two-way ANOVA with a mixed linear model for repeated measures, followed by a Bonferonni's corrected post hoc test for pairwise comparisons at each time point. The differences were considered statistically significant at a $p$ level of $\leq 0.05$.

\section{RESULTS}

Construction of a newly designed shuttle vector $p S$ VJS01 - Plasmid vectors and cloning hosts are critical to successful development of flavivirus infectious clone (Ruggli \& Rice 1999). Many strategies to stabilise flavivirus genome aim to reduce plasmid copy number in E. coli by using low-copy plasmid or BAC vectors. We sought a similar approach to lower plasmid copy number in yeast by using a chromosome-derived origin of replication. The pSVJS01 backbone is based on pBeloBAC11, a BAC vector. To create the new shuttle vector, a $2-\mathrm{kb}$ fragment was amplified by PCR from pRS414 containing CEN6/ARS H1 and TRP1. A T7 promoter recognition site and a MCS were also engineered into this fragment. Plasmid pSVJS01 was assembled by inserting 2-kb fragment into NotI-linearised pBeloBAC11 vector by homologous recombination. Purified plasmid DNA

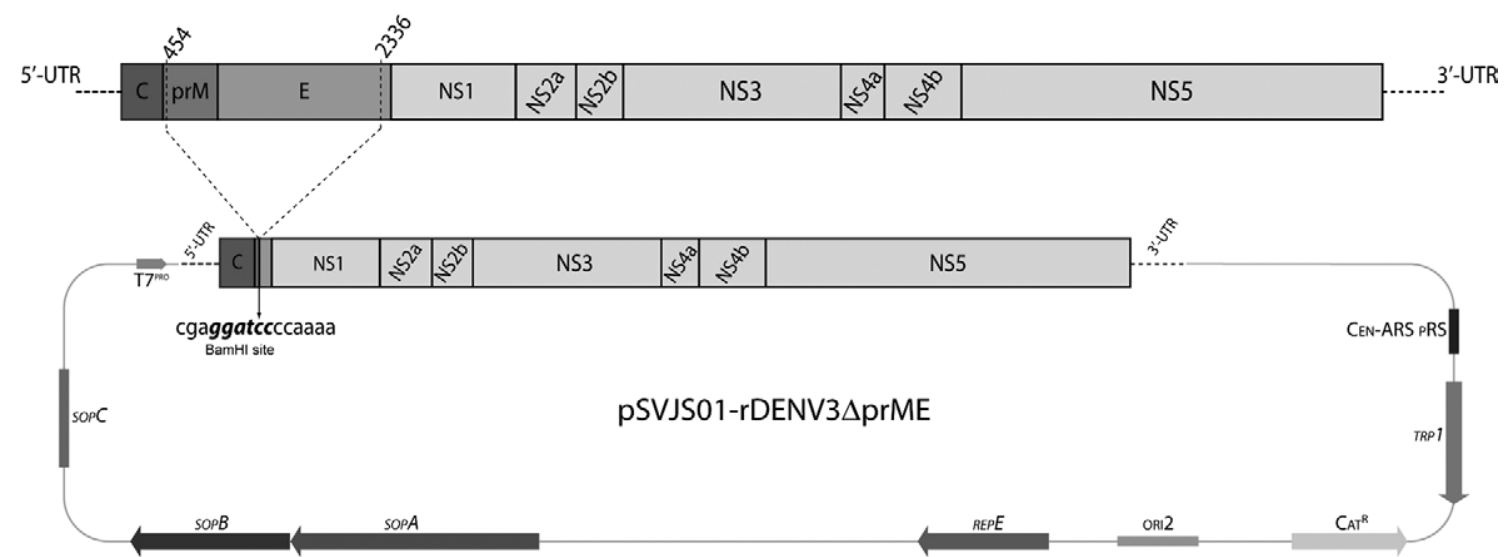

Fig. 2: schematic diagram for the construction of a dengue virus type 3 (DENV-3) replicon with the yeast shuttle vector pSVJS01. DENV-3 replicon was generated by a polymerase chain reaction-mediated deletion into the structural proteins coding region [from nucleotide (nt) 454nt 2336]. DENV-3 replicon was assembled into pSVJS01 vector by homologous recombination, creating pSVJS01-rDENV-3 $\triangle$ prME. A unique BamHI site was inserted into DENV-3 replicon sequence as a genetic marker. NS: nonstructural; UTR: untranslated region. 
was amplified in DH10B E. coli strain and correct cloning of inserts was confirmed by restriction pattern on agarose gel electrophoresis (data not shown).

Assembly of a full-length DENV-3 infectious clone - Fig. 1 shows the cloning strategy used to construct DENV-3 infectious clone. DENV-3 genome was amplified from independent isolated cDNA clones in five overlapping subgenomic fragments. All five fragments required to produce DENV-3 infectious clone along with NotI-linearised pSVJS01 vector were introduced into YPH252 yeast strain by electroporation and a full-length infectious clone (pSVJS01-IC-DENV-3) was assembly by homologous recombination. Sequence analysis confirmed the successful construction of DENV-3 infectious clone and identified one silent mutation at position 9490 (A9490G) in DENV-3 coding region.

Virus rescue and in vitro characterisation of transcript-derived DENV-3 - Full-length PCR amplicons derived from $\mathrm{pSVJS} 01-\mathrm{IC}-\mathrm{DENV}-3$ \#3 were in vitro synthesised and resulting RNA transcripts were electroporated into BHK-21 cells. Cells expressing DENV-3 proteins were easily detected four days after electroporation with DENV-3 full-length transcripts, as demonstrated by IFA (Fig. 3A). Increasing levels of antigen-positive cells were observed over time clearly showing RNA transcripts derived from pSVJS01-IC-DENV-3 \#3 are infectious in BHK-21 cells. On day 8, pronounced signs of cytopathic effect were visible in BHK-21 cell monolayers. In vitro characterisation by focus-forming assay of the rescued virus showed no significant difference between WTDENV-3 (Fig. 4A) and transcript-derived DENV-3 (Fig. 4B) in focus formation and size. Growth kinetics of transcript-derived DENV-3 (Fig. 5B) was also equivalent to WT-DENV-3 (Fig. 5A) at MOI of 0.1 in mosquito C6/36 cells. Transcript-derived DENV-3 grew slightly slowly than WT-DENV-3, but this difference was not statistically significant $(p>0.05$ at each time point). Both viruses reached comparable virus titres on day 5 .
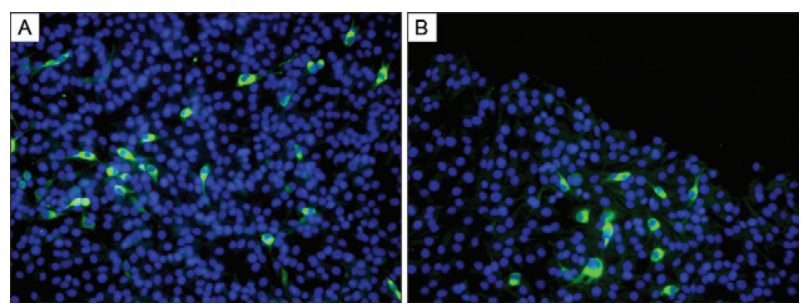

Fig. 3: transcript-derived IC-dengue virus type 3 (DENV-3) \#3 protein expression in BHK-21 cells analysed by immunofluorescence assay. BHK-21 cells were electroporated with in vitro transcribed RNA from IC-DENV-3 \#3 infectious clone. On day 4 after electroporation, cells were fixed and incubated with a polyclonal hyperimmune mouse ascitic fluid raised against group B flaviviruses, followed by an anti-mouse $\mathrm{IgG}$ antibody conjugated to fluorescein isothiocyanate. Cell nuclei were stained with a Hoechst 33258 solution. Images were captured in a DMI 4000B inverted microscope (Leica) with a 200X magnification factor. A: BHK-21 cells electroporated with transcriptderived IC-DENV-3 \#3; B: transcript-derived IC-DENV-3 \#3 remains infectious after 10 repeated passages of the plasmid in yeast.
Stability of the DENV-3 infectious clone in yeast - In order to further characterise DENV-3 infectious clone, the genome stability of functional cDNA clones was examined in yeast. Recombinant clones were grown in drop-out YNB agar plates for 10 subsequent passages separated by three days interval each. No alteration in growth characteristics, colony morphology or coloration was observed during these passages. RNA transcripts derived from DENV-3 infectious clone after repeated passages of the plasmid in yeast remained infectious upon transfection into BHK-21 cells, as shown in Fig. 3B. More importantly, sequence analysis identified no additional mutation in the cloned genome, indicating DENV-3 infectious clone could be stably propagated in yeast for at least 10 passages.

Construction and characterisation of a DENV-3 replicon - DENV-3 replicon was generated by an inframe deletion removing almost the entire prM-E conding region (from nt 454-nt 2336), as illustrated in Fig. 2. Only the first six codons of prM and the last 26 codons of $\mathrm{E}$ were retained in the replicon sequence, as previously described (Pang et al. 2001). DENV-3 replicon was cloned into NotI-linearised pSVJS01 as a set of five fragments by homologous recombination technique in YPH252 yeast strain, generating plasmid pSVJS01rDENV-3 3 prME. As part of the cloning strategy, a Bam$\mathrm{HI}$ site was engineered into pSVJS01-rDENV-3 $\Delta \mathrm{prME}$ sequence to serve as a genetic marker. PCR amplification and digestion by restriction enzyme confirmed the successful deletion of the target structural protein coding region (data not shown). Full-length PCR product derived from pSVJS01-rDENV-3 transcribed and resulting DENV-3 replicon transcript was electroporated in BHK-21 cells. Total RNA was purified and replication of DENV-3 replicon monitored by RT-PCR over time. On day 3 (Fig. 6B, Lane 2), a band corresponding to 1,646-bp in length was amplified by RT-PCR. Band size is coherent with the in-frame deletion in DENV-3 replicon (Fig. 6A). The same band was amplified on day 4 (Fig. 6B, Lane 3), but the intensity of the band was much higher than observed on day 3, showing DENV-3 replicon is functional and capable of autonomous replication in BHK-21 cells. This peak in
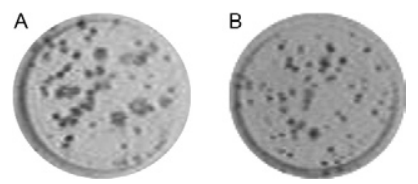

Fig. 4: in vitro characterisation by focus-forming assay. Mosquito C6/36 cells were infected with either wild-type dengue virus type 3 (WT-DENV-3) or transcript-derived IC-DENV-3 \#3. After removing the inocula, cells were covered with overlay medium and incubated for five days. Cells were fixed and incubated with a polyclonal hyperimmune mouse ascitic fluid raised against group B flaviviruses, followed by a horseradish peroxidase-conjugated recombinant protein $\mathrm{G}$. Foci were developed by adding 3-amino-9-ethylcarbazole substrate. A: WT-DENV-3 foci in mosquito C6/36 cells; B: transcript-derived IC-DENV-3 \#3 foci in mosquito C6/36 cells. 
replication of DENV-3 replicon on day 4 is consistent with published results in the literature (Alcaraz-Estrada et al. 2010). However, replication of DENV-3 replicon is transient and no PCR amplification was observed on day 10 (Fig 6B, Lane 4). Digestion of RT-PCR product from day 4 with $B a m \mathrm{HI}$ (Lane 5) generated fragments of 1,192-bp and 454-bp, confirming the genetic marker was retained in DENV-3 replicon.

\section{DISCUSSION}

Flaviviruses research has experienced a remarkable progress by the advent of reverse genetics systems in the past decades. However, obtaining flavivirus infectious clones has not been an easy task. The single most reported hurdle in the establishment of these systems is an inherent instability of flavivirus genome in E. coli $(\mathrm{Pu}$ et al. 2011). In this paper, the successful construction and characterisation of a complete reverse genetics system for DENV-3 has been described. This system was developed with a low passage DENV-3 clinical isolate. Infectious cDNA clone methodology has been developed for all
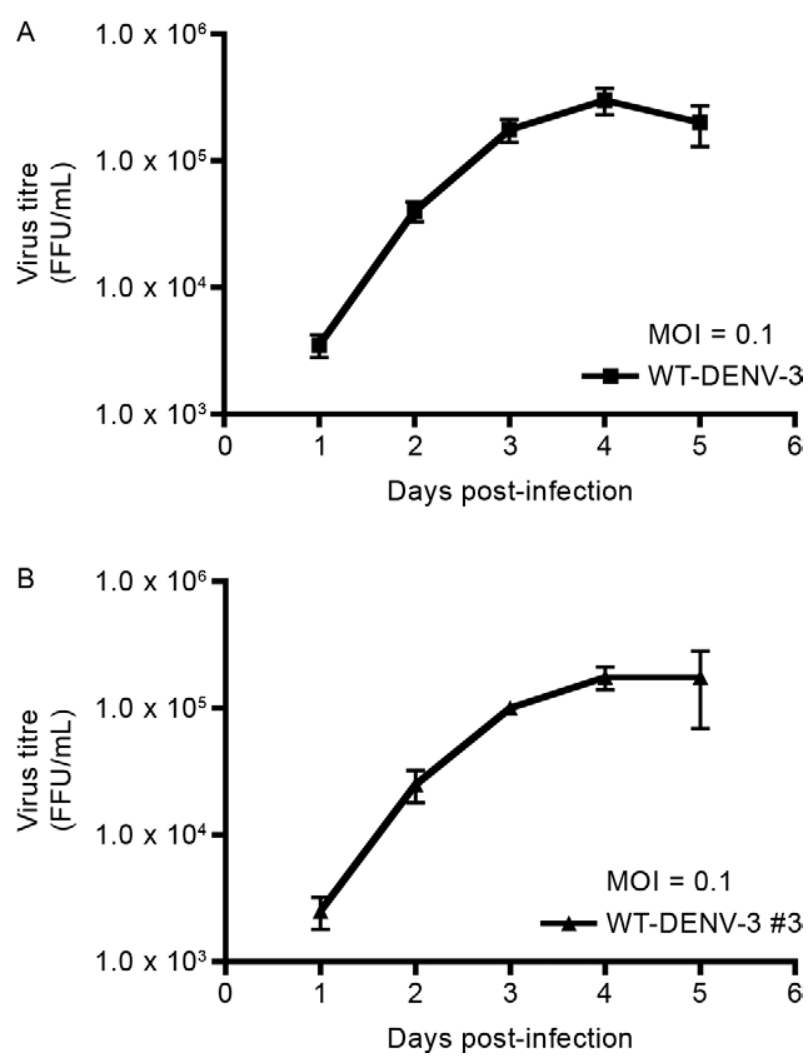

Fig. 5: transcript-derived IC-dengue virus type 3 (DENV-3) \#3 and wild-type (WT)-DENV-3 growth kinetics in mosquito C6/36 cells. Mosquito C6/36 cells were infected in duplicate at multiplicity of infection (MOI) of 0.1 with WT-DENV-3 or transcript-derived DENV-3. A sample of the supernatant of infected cells was collected daily and virus titres were determined by titration in mosquito $\mathrm{C} 6 / 36$ cells. No statistically significant difference in virus titre $(p>0.05)$ was found between the viruses at different time points. A: WT-DENV-3 growth curve in mosquito C6/36 cells; B: transcript-derived IC-DENV-3 \#3 growth curve in mosquito $\mathrm{C6} / 36$ cells; FFU: focus-forming unit. four DENV serotypes and other major flaviviruses, but most systems utilised established strains or laboratoryadapted clinical isolates as parental virus. Even though the value of these studies is unquestionable, it is important to point out the need to expand this methodology for low passage clinical isolates to gain a complete picture in flavivirus biology, particularly in virus pathogenesis, virulence determinants and vector competence (Ubol et al. 2008, Tuiskunen et al. 2011, Cox et al. 2012).

Our strategy employed the use of homologous recombination in yeast cells to assemble DENV-3 infectious clone and replicon. Yeast strains are versatile and convenient cloning hosts, frequently used in the construction of reverse genetics systems for DENV (Polo et al. 1997, Puri et al. 2000, Pang et al. 2001, Pu et al. 2011), because they are more tolerant to unstable sequence than bacteria. Homologous recombination in yeast is also a simple and robust method with higher cloning efficiency when compared to standard cloning procedures in $E$.

\section{A}

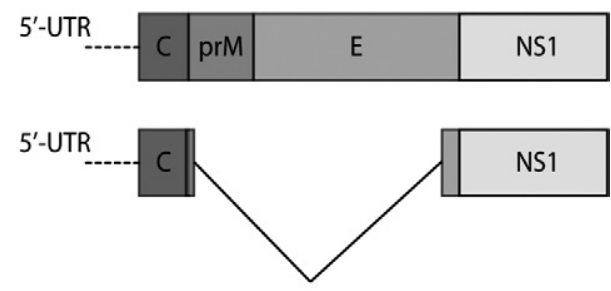

B

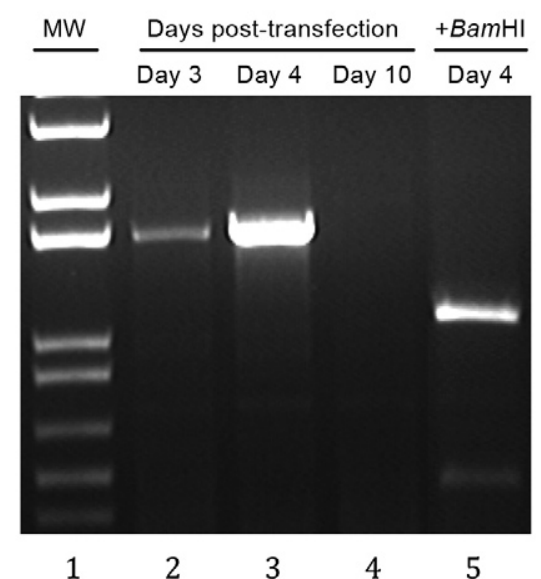

Fig. 6: kinetics of dengue virus type 3 (DENV-3) replicon RNA synthesis in transfected BHK-21 cell. A unique BamHI site was inserted into DENV-3 replicon sequence during molecular cloning and used as a genetic marker. A: schematic diagram of rDENV-3 $\Delta$ prME in-frame deletion and genetic marker analysis; $\mathrm{B}$ : at different time points, total RNA was purified from BHK-21 cells transfected with rDENV$3 \Delta$ prME replicon. An 1,646-bp fragment spanning the BamHI site was amplified by reverse transcription-polymerase chain reaction (RT-PCR) on day 3 (Lane 2) and 4 (Lane 3) and analysed on agarose gel electrophoresis. The RT-PCR product on day 4 was digested with BamHI (Lane 5) generating fragments of 1,192-bp and 454-bp. No amplification was observed on day 10 (Lane 4). An $1 \mathrm{~Kb}$ plus DNA ladder (Invitrogen) was loaded on Lane 1; MW: molecular weight; NS: nonstructural; UTR: untranslated region. 
coli (Gibson 2009, Shanks et al. 2009). We have also demonstrated the utility of pSVJS01 vector for stable propagation of DENV-3 genome in yeast. Several reverse genetics systems were established using low-copy plasmid or BAC vectors in an effort to lower plasmid copy number in E. coli and, as consequence, achieve stability of cloned genomes. In a previous attempt, we were only able to recovery a DENV-3 infectious clone by an in vitro ligation strategy using the pBSC shuttle vector (JJS Santos \& LHVG Gil, unpublished observations). Despite this vector has been successfully used to manipulate pestivirus genomes (Ansari et al. 2004, Gil et al. 2006), pBSC vector carries a $2 \mu \mathrm{m}$ high copy number origin of replication. We hypothesised that reducing plasmid copy number in yeast using a chromosome-derived origin of replication would allow the successful assembly of a full-length DENV-3 infectious clone.

In contrast with $\mathrm{Pu}$ et al. (2011), no silent mutations were necessary to be introduced to circumvent sequence instability and recovery a functional infectious clone. RNA transcripts derived from DENV-3 infectious clone were infectious upon transfection into BHK-21 cells. Transcript-derived DENV-3 exhibited growth kinetics, focus formation and size comparable to WT-DENV-3 in mosquito C6/36 cell culture. Of paramount importance, RNA transcripts remained infectious even after repeated passages of the plasmid in yeast. The long-term stability of many infectious clones previously described remains largely undetermined after serial passages of the cloned genome in the harbouring host. Only few reports have more closely investigated this stability of continuing propagated flavivirus infectious clones (Hurrelbrink et al. 1999, Bredenbeek et al. 2003, Pu et al. 2011). Most of their assessment relies on virus rescue from or restriction pattern of passaged plasmid. Our work went one step further showing not only the ability of rescuing virus, but also that the nucleotide sequence of DENV-3 viral genome remain unchanged after successive passages of the plasmid in yeast.

Different approaches have been used for the construction of flavivirus replicons. We used an approach previously described for a DENV-2 replicon by selectively removing almost the entire prM-E coding region (Pang et al. 2001). DENV-3 replicon was also easily assembled into pSVJS01 shuttle vector. In vitro characterisation of DENV-3 replicon confirmed its ability to replicate transiently in BHK-21 cells.

In summary, we describe an alternative and effective strategy to bypass flavivirus genome instability commonly observed in E. coli by using homologous recombination in yeast and a newly designed pSVJS01 vector. This reverse genetics system will be a valuable tool for studying the molecular mechanisms involved in viral genome replication, virion assembly, virus pathogenesis and vaccine development. This approach can be applied to others DENV serotypes or flaviviruses.

\section{ACKNOWLEDGEMENTS}

To PDTIS-FIOCRUZ, for the use of its facilities, and to Dr Pedro Vasconcelos, Evandro Chagas Institute (Belém, state of Pará, Brazil), for kindly supplying the polyclonal hyperimmune ascitic fluid.

\section{REFERENCES}

Alcaraz-Estrada SL, Manzano MI, del Angel RM, Levis R, Padmanabhan R 2010. Construction of a dengue virus type 4 reporter replicon and analysis of temperature-sensitive mutations in nonstructural proteins 3 and 5. J Gen Virol 91: 2713-2718.

Alvarez DE, Lodeiro MF, Luduena SJ, Pietrasanta LI, Gamarnik AV 2005. Long-range RNA-RNA interactions circularize the dengue virus genome. J Virol 79: 6631-6643.

Ansari IH, Chen LM, Liang D, Gil LH, Zhong W, Donis RO 2004. Involvement of a bovine viral diarrhea virus NS5B locus in virion assembly. J Virol 78: 9612-9623.

Blaney Jr JE, Hanson CT, Firestone CY, Hanley KA, Murphy BR, Whitehead SS 2004. Genetically modified, live attenuated dengue virus type 3 vaccine candidates. Am J Trop Med Hyg 71: 811-821.

Bouloy M, Flick R 2009. Reverse genetics technology for Rift Valley fever virus: current and future applications for the development of therapeutics and vaccines. Antiviral Res 84: 101-118.

Bredenbeek PJ, Kooi EA, Lindenbach B, Huijkman N, Rice CM, Spaan WJ 2003. A stable full-length yellow fever virus cDNA clone and the role of conserved RNA elements in flavivirus replication. J Gen Virol 84: 1261-1268.

Chambers TJ, Hahn CS, Galler R, Rice CM 1990. Flavivirus genome organization, expression and replication. Annu Rev Microbiol 44: 649-688.

Clyde K, Kyle JL, Harris E 2006. Recent advances in deciphering viral and host determinants of dengue virus replication and pathogenesis. J Virol 80: 11418-11431.

Cordeiro MT, Schatzmayr HG, Nogueira RM, Oliveira VF, Melo WT, Carvalho EF 2007. Dengue and dengue hemorrhagic fever in the state of Pernambuco, 1995-2006. Rev Soc Bras Med Trop 40: 605-611.

Cox J, Mota J, Sukupolvi-Petty S, Diamond MS, Rico-Hesse R 2012. Mosquito bite delivery of dengue virus enhances immunogenicity and pathogenesis in humanized mice. $J$ Virol 86: 7637-7649.

Das S, Garver L, Ramirez JR, Xi Z, Dimopoulos G 2007. Protocol for dengue infections in mosquitoes (Ae. aegypti) and infection phenotype determination. J Vis Exp 2007: 220.

Fernandez-Garcia MD, Mazzon M, Jacobs M, Amara A 2009. Pathogenesis of flavivirus infections: using and abusing the host cell. Cell Host Microbe 5: 318-328.

Finley Jr RL, Brent R 1994. Interaction mating reveals binary and ternary connections between Drosophila cell cycle regulators. Proc Natl Acad Sci USA 91: 12980-12984.

Gibson DG 2009. Synthesis of DNA fragments in yeast by one-step assembly of overlapping oligonucleotides. Nucleic Acids Res 37: 6984-6990.

Gil LH, Ansari IH, Vassilev V, Liang D, Lai VC, Zhong W, Hong Z, Dubovi EJ, Donis RO 2006. The amino-terminal domain of bovine viral diarrhea virus Npro protein is necessary for alpha/beta interferon antagonism. $J$ Virol 80: 900-911.

Guy B, Nougarede N, Begue S, Sanchez V, Souag N, Carre M, Chambonneau L, Morrisson DN, Shaw D, Qiao M, Dumas R, Lang J, Forrat R 2008. Cell-mediated immunity induced by chimeric tetravalent dengue vaccine in naïve or flavivirus-primed subjects. Vaccine 26: 5712-5721.

Hurrelbrink RJ, Nestorowicz A, McMinn PC 1999. Characterization of infectious Murray Valley encephalitis virus derived from a stably cloned genome-length cDNA. J Gen Virol 80: 3115-3125.

Jones CT, Patkar CG, Kuhn RJ 2005. Construction and applications of yellow fever virus replicons. Virology 331: 247-259. 
Kapoor M, Zhang L, Mohan PM, Padmanabhan R 1995. Synthesis and characterization of an infectious dengue virus type-2 RNA genome (New Guinea C strain). Gene 162: 175-180.

Khromykh AA, Westaway EG 1997. Subgenomic replicons of the flavivirus Kunjin: construction and applications. J Virol 71: 1497-1505.

Kurosu T, Khamlert C, Phanthanawiboon S, Ikuta K, Anantapreecha S 2010. Highly efficient rescue of dengue virus using a co-culture system with mosquito/mammalian cells. Biochem Biophys Res Commun 394: 398-404.

Lai CJ, Zhao BT, Hori H, Bray M 1991. Infectious RNA transcribed from stably cloned full-length cDNA of dengue type 4 virus. Proc Natl Acad Sci USA 88: 5139-5143.

Lindenbach BD, Thiel HJ, Rice CM 2007. Flaviviruses: the virus and their replication. In DM Knipe, PM Hpwley, Fields virology, 5th ed., Vol. 1, Lippincott Williams \& Wilkins, Philadelphia, p. 1102-1152.

Liu WJ, Wang XJ, Clark DC, Lobigs M, Hall RA, Khromykh AA 2006. A single amino acid substitution in the West Nile virus nonstructural protein NS2A disables its ability to inhibit alpha/ beta interferon induction and attenuates virus virulence in mice. J Virol 80: 2396-2404.

Meilhoc E, Masson JM, Teissie J 1990. High efficiency transformation of intact yeast cells by electric field pulses. Nat Biotechnol 8: 223-227.

Messer WB, Yount B, Hacker KE, Donaldson EF, Huynh JP, de Silva AM, Baric RS 2012. Development and characterization of a reverse genetic system for studying dengue virus serotype 3 strain variation and neutralization. PLoS Negl Trop Dis 6: e1486.

Monath TP 1994. Dengue: the risk to developed and developing countries. Proc Natl Acad Sci USA 91: 2395-2400.

Pang X, Zhang M, Dayton AI 2001. Development of dengue virus type 2 replicons capable of prolonged expression in host cells. BMC Microbiol 1: 18.

Polo S, Ketner G, Levis R, Falgout B 1997. Infectious RNA transcripts from full-length dengue virus type 2 cDNA clones made in yeast. J Virol 71: 5366-5374.

Pu SY, Wu RH, Yang CC, Jao TM, Tsai MH, Wang JC, Lin HM, Chao YS, Yueh A 2011. Successful propagation of flavivirus infectious cDNAs by a novel method to reduce the cryptic bacterial promoter activity of virus genomes. J Virol 85: 2927-2941.

Puig-Basagoiti F, Deas TS, Ren P, Tilgner M, Ferguson DM, Shi PY 2005. High-throughput assays using a luciferase-expressing repli- con, virus-like particles and full-length virus for West Nile virus drug discovery. Antimicrob Agents Chemother 49: 4980-4988.

Puri B, Polo S, Hayes CG, Falgout B 2000. Construction of a full length infectious clone for dengue-1 virus Western Pacific, 74 strain. Virus Genes 20: 57-63.

Rice CM, Grakoui A, Galler R, Chambers TJ 1989. Transcription of infectious yellow fever RNA from full-length cDNA templates produced by in vitro ligation. New Biol 1: 285-296.

Ruggli N, Rice CM 1999. Functional cDNA clones of the Flaviviridae: strategies and applications. Adv Virus Res 53: 183-207.

Sambrook J, Russell DW 2001. Molecular cloning: a laboratory manual, 3rd ed., Cold Spring Harbor Laboratory Press, New York, 2344 pp.

Shanks RM, Kadouri DE, MacEachran DP, O’Toole GA 2009. New yeast recombineering tools for bacteria. Plasmid 62: 88-97.

Sikorski RS, Hieter P 1989. A system of shuttle vectors and yeast host strains designed for efficient manipulation of DNA in Saccharomyces cerevisiae. Genetics 122: 19-27.

Sriburi R, Keelapang P, Duangchinda T, Pruksakorn S, Maneekarn N, Malasit P, Sittisombut N 2001. Construction of infectious dengue 2 virus cDNA clones using high copy number plasmid. $J$ Virol Methods 92: 71-82.

Suzuki R, de Borba L, dos Santos CND, Mason PW 2007. Construction of an infectious cDNA clone for a Brazilian prototype strain of dengue virus type 1: characterization of a temperature-sensitive mutation in NS1. Virology 362: 374-383.

Tuiskunen A, Wahlstrom M, Bergstrom J, Buchy P, Leparc-Goffart I, Lundkvist A 2011. Phenotypic characterization of patient dengue virus isolates in BALB/c mice differentiates dengue fever and dengue hemorrhagic fever from dengue shock syndrome. Virol J 8: 398 .

Ubol S, Chareonsirisuthigul T, Kasisith J, Klungthong C 2008. Clinical isolates of dengue virus with distinctive susceptibility to nitric oxide radical induce differential gene responses in THP-1 cells. Virology 376: 290-296.

Widman DG, Ishikawa T, Fayzulin R, Bourne N, Mason PW 2008. Construction and characterization of a second-generation pseudoinfectious West Nile virus vaccine propagated using a new cultivation system. Vaccine 26: 2762-2771.

Yamshchikov VF, Wengler G, Perelygin AA, Brinton MA, Compans RW 2001. An infectious clone of the West Nile flavivirus. Virology 281: 294-304. 